\title{
OPERATIONS PRESERVING THE PFAFFIAN PROPERTY OF A GRAPH
}

\author{
C. H. C. LITTLE and F. RENDL
}

(Received 8 May 1989; revised 2 March 1990)

Communicated by L. Caccetta

\begin{abstract}
Pfaffian graphs are those which can be oriented so that the 1-factors have equal sign, as calculated according to the prescription of Kasteleyn. We consider various operations on graphs and examine their effect on the Pfaffian property. We show that the study of Pfaffian graphs may be reduced to the case of subcubic graphs (graphs in which no vertex has degree greater than 3 ) or bricks (3-connected bicritical graphs).
\end{abstract}

1980 Mathematics subject classification (Amer. Math. Soc.) (1985 Revision): 05 C 70.

\section{Pfaffian graphs}

In this paper we consider only finite graphs with no loops or multiple edges.

A method for the enumeration of the 1-factors of a planar graph $G$ has been given by Kasteleyn [1]. In order to explain Kasteleyn's idea, let us first transform $G$ into a directed graph $G^{*}$ by assigning an orientation to each edge. Consider the set $F=\left\{f_{1}, \ldots, f_{k}\right\}$ of 1-factors of $G^{*}$. For all $i$ write

$$
f_{i}=\left\{\left(u_{i 1}, w_{i 1}\right),\left(u_{i 2}, w_{i 2}\right), \ldots,\left(u_{i n}, w_{i n}\right)\right\},
$$

where $u_{i j}, w_{i j} \in V\left(G^{*}\right)$ for all $j$. (We write $(u, w)$ for an edge of $G^{*}$ directed from vertex $u$ to vertex $w$.) Associate with $f_{i}$ a plus sign if

$$
u_{i 1} w_{i 1} u_{i 2} w_{i 2} \cdots u_{i n} w_{i n}
$$

is an even permutation of

$$
u_{11} w_{11} u_{12} w_{12} \cdots u_{1 n} w_{1 n},
$$

(C) 1991 Australian Mathematical Society 0263-6115/91 \$A2.00+0.00 
and a minus sign otherwise. Thus the signs of the 1-factors are dependent on the choice of $f_{1}$, but it is clear that the resulting partition of $F$ into two complementary subsets is not. Moreover a permutation of the edges in a particular 1-factor leaves the sign of that 1-factor unchanged, but reversal of the orientation of an edge $e$ alters the sign of each 1 -factor containing $e$. We say that $G$ is Pfaffian if it is possible to choose $G^{*}$ so that its 1 -factors agree in sign. In this case we call $G^{*}$ a Pfaffian orientation of $G$.

Kasteleyn [1] shows that once a Pfaffian orientation for $G$ has been found, then the enumeration of the 1 -factors of $G$ is reduced to the evaluation of a certain determinant. Moreover he gives an efficient algorithm for the construction of a Pfaffian orientation for any planar graph.

Though every planar graph is Pfaffian, many non-planar graphs are not. The following condition for a graph to be Pfaffian is given in [2] (see also [6, page 324]). A set $S$ of 1-factors in a graph $G$ with orientation $G^{*}$ is intractable if

(a) each edge of $G$ belongs to an even number of 1 -factors in $S$, and

(b) an odd number of 1-factors in $S$ have a plus sign (or a minus sign). Clearly (a) implies that $|S|$ is even, and that property (b) is independent of the choice of orientation. A necessary and sufficient condition for $G$ to be Pfaffian is for it to have no intractable set of 1 -factors.

Figure 1 gives three examples of non-Pfaffian graphs. For each example, we list the 1-factors of an intractable set, with their signs as determined by the orientation and the choice of $f_{1}$. (Here, and subsequently, a 1-factor $\left\{\left(u_{1}, w_{1}\right),\left(u_{2}, w_{2}\right), \ldots,\left(u_{n}, w_{n}\right)\right\} \quad$ is abbreviated as $\left(u_{1} w_{1}\right)\left(u_{2} w_{2}\right) \ldots$ $\left.\left(u_{n} w_{n}\right).\right)$

Examples such as these motivate the following conjecture.

CONJECTURE 1. In any non-Pfaffian graph the cardinality of a minimum intractable set of 1-factors is 6 .

A second criterion for a graph to be Pfaffian involves alternating circuits. We identify paths and circuits with their edge sets. A circuit is alternating with respect to two given 1-factors if it is their symmetric difference. The symmetric difference of two arbitrary 1 -factors is the union of a set of vertex disjoint alternating circuits.

An alternating circuit in a directed graph is called clockwise odd or clockwise even according to the parity of the number of its edges directed in the clockwise sense. Since every alternating circuit has even length, it does not matter which sense is designated as clockwise. It was shown by Kasteleyn [1] that two 1-factors agree in sign if and only if the number of clockwise even 
alternating circuits included in their symmetric difference is even. It follows that an orientation of a graph is Pfaffian if and only if every alternating circuit is clockwise odd. In fact it suffices to check this condition only for those circuits which are alternating with respect to an arbitrary but fixed 1-factor.

A set $S$ of alternating circuits in a graph $G$ with orientation $G^{*}$ is called intractable if (a) each edge of $G$ belongs to an even number of alternating circuits in $S$, and (b) an odd number of alternating circuits in $S$ are clockwise even.

Property (a) implies that property (b) is independent of the choice of orientation.

It is shown in [2] that $G$ is Pfaffian if and only if it has no intractable set of alternating circuits. For instance, in the graphs of Figure $1,\left\{f_{1}+f_{2}, f_{3}+\right.$ $\left.f_{4}, f_{5}+f_{6}\right\}$ forms an intractable set of alternating circuits.

The following conjecture is closely related to Conjecture 1 .

CONJECTURE 2. In any non-Pfaffian graph the cardinality of a minimum intractable set of alternating circuits is 3 .

Certain operations preserve the property of being Pfaffian or non-Pfaffian. An easy example is even subdivision. We define a subdivision of a graph $G$ to be a graph obtained from $G$ by replacing an edge joining vertices $v$ and $w$ by a path $P$ joining $v$ and $w$ such that $V(P) \cap V(G)=\{v, w\}$. The subdivision is called even if $|V(P)|$ is even. A graph $G^{\prime}$ is called an even subdivision of $G$ if for some positive integer $k$ there exist graphs $G_{1}, G_{2}, \ldots, G_{k}$ such that $G_{1}=G, G_{k}=G^{\prime}$ and, for all integers $i$ such that $1 \leq i<k$, $G_{i+1}$ is an even subdivision of $G_{i}$.

It is easy to see that if $G^{\prime}$ is an even subdivision of $G$ then $G^{\prime}$ is Pfaffian if and only if $G$ is Pfaffian. Indeed, suppose that $(x, y)$ is a directed edge in some orientation of $G$, to be replaced by directed edges $(x, a),(a, b)$, $(b, y)$ in an orientation of $G^{\prime}$, and that the remaining edges of $G$ and $G^{\prime}$ are identical and agree in orientation. Then 1-factors in $G$ are transformed into 1 -factors in $G^{\prime}$ either by adjoining $(a b)$ or by replacing $(x y)$ by $(x a)(b y)$. Since $G^{\prime}$ has no other 1 -factors, the result is immediate.

Sections 2 and 3 examine the effect on the Pfaffian or non-Pfaffian property of some other operations on a graph. Both of these sections reduce the problem of characterising Pfaffian graphs to the case of a special kind of graph, a subcubic graph in Section 2 and a brick in Section 3. 


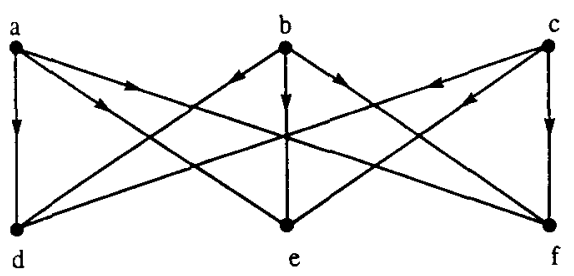

$\begin{array}{lll}f_{1}(a d)(b e)(c f) & + \\ f_{2}(a d)(b f)(c e) & - \\ f_{3}(a e)(b f)(c d) & + \\ f_{4}(a e)(b d)(c f) & - \\ f_{5}(a f)(b d)(c e) & + \\ f_{6}(a f)(b e)(c d) & -\end{array}$

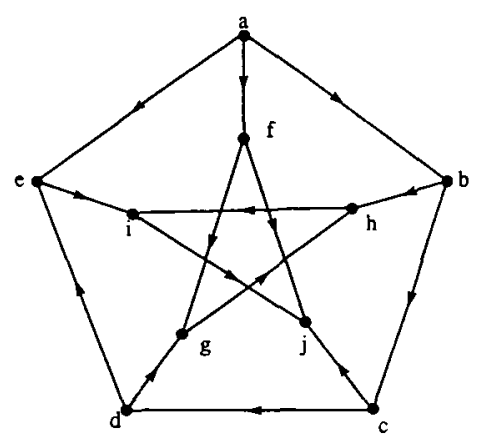

$\begin{array}{lll}f_{1} & (a f)(b h)(c j)(d g)(e i) & + \\ f_{2} & (a f)(b c)(d e)(g h)(i j) & - \\ f_{3} & (a e)(b h)(c d)(f g)(i j) & + \\ f_{4} & (a b)(d e)(c j)(f g)(h i) & - \\ f_{5} & (a e)(b c)(d g)(f j)(h i) & - \\ f_{6} & (a b)(c d)(e i)(f j)(g h) & +\end{array}$

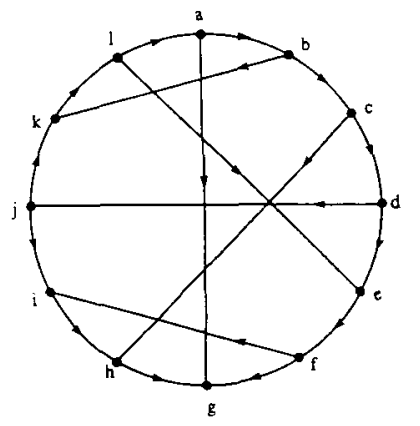

$f_{1}(a b)(c d)(j k)(l e)(f i)(h g)+$

$\begin{array}{ll}f_{2} & (b c)(d j)(k l)(e f)(i h)(a g)+\end{array}$

$f_{3}(d j)(l e)(f i)(a g)(b k)(c h) \quad-$

$f_{4}(a b)(k l)(f g)(j i)(c h)(d e)-$

$f_{5}(b c)(j k)(i h)(f g)(d e)(l a)+$

$\begin{array}{ll}f_{6} & (c d)(e f)(h g)(j i)(b k)(l a) \quad-\end{array}$

FIGURE 1. Some non-Pfaffian graphs

\section{Reduction to subcubic graphs}

We call a graph subcubic if no vertex has degree greater than 3 . In this section we show that the problem of characterising Pfaffian graphs may be reduce to the case of subcubic graphs.

Let $v$ be a vertex of degree $k>3$ in a graph $G$. Let $w_{1}, w_{2}, \ldots, w_{k}$ 

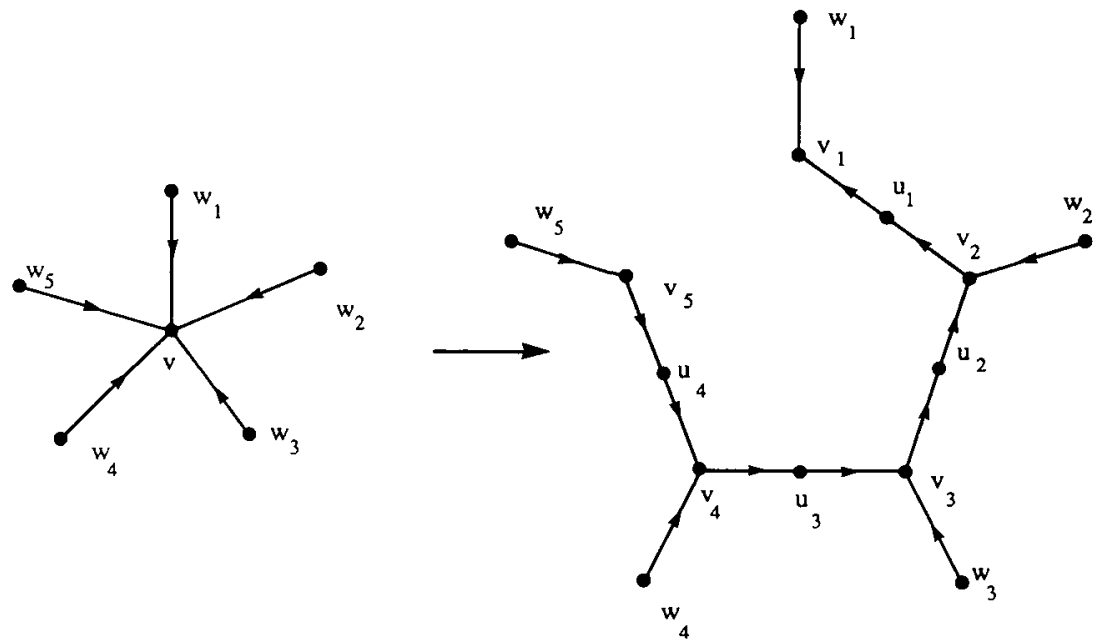

FIGURE 2. Reduction to a subcubic graph

be the neighbours of $v$, and without loss of generality let $G$ be oriented so that $v$ is a sink. Let $G^{\prime}$ be the directed graph obtained from $G$ by replacing $v$ and its incident edges by new vertices

$$
v_{1}, v_{2}, \ldots, v_{k}, u_{1}, u_{2}, \ldots, u_{k-1}
$$

and directed edges

$$
\begin{aligned}
&\left(w_{1}, v_{1}\right),\left(w_{2}, v_{2}\right), \ldots,\left(w_{k}, v_{k}\right),\left(u_{1}, v_{1}\right),\left(u_{2}, v_{2}\right), \ldots, \\
&\left(u_{k-1}, v_{k-1}\right),\left(v_{2}, u_{1}\right),\left(v_{3}, u_{2}\right), \ldots,\left(v_{k}, u_{k-1}\right) .
\end{aligned}
$$

(See Figure 2 for the case $k=5$.) Each 1-factor of $G$ is of the form $A\left(w_{i} v\right)$ for some $i$, where $A \subset E(G)$, and corresponds to the 1-factor

$$
A\left(w_{i} v_{i}\right)\left(u_{i-1} v_{i-1}\right)\left(u_{i-2} v_{i-2}\right) \cdots\left(u_{1} v_{1}\right)\left(v_{i+1} u_{i}\right)\left(v_{i+2} u_{i+1}\right) \cdots\left(v_{k} u_{k-1}\right)
$$

in $G^{\prime}$. Then $G^{\prime}$ has no 1 -factors other than those that correspond to 1-factors of $G$ in this way. Since

$$
v_{i} u_{i-1} v_{i-1} u_{i-2} v_{i-2} \cdots u_{1} v_{1} v_{i+1} u_{i} v_{i+2} u_{i+1} \cdots v_{k} u_{k-1}
$$

is an even permutation of

$$
v_{i-1} u_{i-2} v_{i-2} u_{i-3} v_{i-3} \cdots u_{1} v_{1} v_{i} u_{i-1} v_{i+1} u_{i} \cdots v_{k} u_{k-1}
$$

for each $i>1$, it follows inductively that the former is an even permutation of

$$
v_{1} v_{2} u_{1} v_{3} u_{2} \cdots v_{k} u_{k-1}
$$

for each $i$. It should now be clear that $G^{\prime}$ is Pfaffian if and only if $G$ is Pfaffian. 


\section{Reduction to bricks}

A graph $G$ is bicritical if $G-\{u, v\}$ has a 1 -factor for any pair of vertices $u$ and $v$. We define a brick to be a 3-connected bicritical graph.

A graph is called 1-factor covered if for each edge $e$ there is a 1-factor containing $e$.

Suppose that $G$ is a graph which is 1 -factor covered but not bicritical. Then (see [4]) it has a maximal set $S$ of vertices such that $|S| \geq 2$ and $G-S$ has exactly $|S|$ odd components (components with an odd number of vertices). Let $|S|=k$, and let $H_{1}, \ldots, H_{k}$ be the components of $G-S$. For each $i$ let $G_{i}$ be the graph obtained from $G$ by contraction of the subgraph $G-V\left(H_{i}\right)$. Let $G_{0}$ be the bipartite graph obtained from $G$ by the successive contraction of $H_{i}$ for each $i$. We call $G_{0}$ the frame. It is shown in [3] that $G_{0}, G_{1}, \ldots, G_{k}$ are 1-factor covered. It is also clear that, for any $i$, the graph obtained from $G$ by the contraction of $H_{i}$ is 1-factor covered.

Now we discard $G_{0}$ and any of $G_{1}, \ldots, G_{k}$ that are isomorphic to $K_{2}$, and we file those of $G_{1}, \ldots, G_{k}$ that are bicritical. The procedure is then repeated recursively for the remaining graphs. Eventually a family of bicritical graphs is obtained.

A bicritical graph may be decomposed into bricks as follows. Let $G$ be bicritical and connected but not a brick. It follows that the connectivity of $G$ is 2 . Hence there are vertices $u, v$ such that $G-\{u, v\}$ is not connected. Let $G_{1}^{\prime}, G_{2}^{\prime}, \ldots, G_{l}^{\prime}$ be the components of $G-\{u, v\}$, and for each $i$ let $G_{i}$ be the graph obtained from $G\left[V\left(G_{i}^{\prime}\right) \cup\{u, v\}\right]$ by adjoining an edge between $u$ and $v$ if they are not already adjacent. It is shown in [5] that $G_{i}$ is bicritical. This procedure is repeated recursively until a list of bricks is obtained.

The brick decomposition procedure described above motivates the study of two operations. Firstly, let $v$ and $w$ be vertices, of equal degree $d$, in graphs $H$ and $K$ respectively. Let $v_{1}, \ldots, v_{d}$ be the neighbours of $v$ and $w_{1}, \ldots, w_{d}$ those of $w$. Let $G$ be the graph obtained from $(H-\{v\}) \cup$ $(K-\{w\})$ by adjoining an edge between $v_{i}$ and $w_{i}$ for each $i$. Then we say that $G$ is formed by splicing $H$ and $K$ at $v$ and $w$ respectively. We call $E(G)-[E(H) \cup E(K)]$ the splice of $G$.

Secondly, let $x$ and $y$ be edges in graphs $H$ and $K$ respectively. Let $L$ be a graph obtained from $H$ and $K$ by identifying $x$ and $y$ to form an edge $e$. Then graphs $L$ and $L-\{e\}$ are said to be obtained from $H$ and $K$ by gluing $H$ and $K$ at $x$ and $y$. The brick decomposition procedure shows that any 1 -factor covered graph may be constructed from bricks by gluing and splicing, where the graphs being glued and spliced each have more than one edge. 
First we show that splicing or gluing a non-Pfaffian graph to another graph yields a non-Pfaffian graph.

THEOREM 1. Let $H, K$ be 1-factor covered graphs spliced at vertices $v$, $w$ respectively to form $G$. If either $H$ or $K$ is non-Pfaffian, then so is $G$.

Proof. Suppose without loss of generality that $H$ is non-Pfaffian. Then $H$ has an intractable set $S$ of 1 -factors. Let $\left\{e_{1}, e_{2}, \ldots, e_{k}\right\}$ be the splice of $G$. For each $i$, let $e_{i}$ join vertices $v_{i} \in V(H)$ and $w_{i} \in V(K)$, and let $S_{i}$ be the set of 1-factors in $S$ which contain the edge $e_{i}^{H}$ of $E(H)$ joining $v_{i}$ and $v$. Since $K$ is 1 -factor covered, there exists a 1 -factor $f_{i}$ in $K$ which contains the edge $e_{i}^{K}$ of $E(K)$ joining $w_{i}$ and $w$. Let $S_{i}^{\prime}$ be the set of 1 -factors of $G$ of the form $\left(s_{i}-\left\{e_{i}^{H}\right\}\right) \cup\left(f_{i}-\left\{e_{i}^{K}\right\}\right) \cup\left\{e_{i}\right\}$, where $s_{i} \in S_{i}$. Then $\bigcup_{i=1}^{k} S_{i}^{\prime}$ is an intractable set of 1 -factors of $G$.

THEOREM 2. Let $H, K$ be connected 1-factor covered graphs, distinct from $K_{2}$, which are glued at edges $x, y$ respectively to form a graph $G$. If either $H$ or $K$ is non-Pfaffian, then so is $G$.

Proof. Suppose without loss of generality that $H$ is non-Pfaffian. Then $H$ has an intractable set $S$ of 1 -factors. Let $u, v$ be the vertices of $V(G)$ that are joined by $x$ in $H$ and by $y$ in $K$. Let $S_{0}$ be the set of 1 -factors in $S$ that contain $x$. Let $S_{1}=S-S_{0}$. Since $K$ is 1 -factor covered, $K-\{y\}$ has a 1 -factor $f_{0}$, and $K-\{u, v\}$ has a 1 -factor $f_{1}$. For each $i \in\{0,1\}$ let $T_{i}$ be the set of 1-factors of $G$ of the form $(s-\{x\}) \cup f_{i}$, where $s \in S_{i}$. Then $T_{0} \cup T_{1}$ is intractable in $G$.

Next let us show that gluing Pfaffian graphs yields a Pfaffian graph.

THEOREM 3. Let $H, K$ be 1-factor covered graphs, glued at edges $x, y$ respectively to form a graph $G$. If $H$ and $K$ are Pfaffian, then so is $G$.

Proof. Let $H, K$ be given orientations so that every alternating circuit is clockwise odd and the orientations coincide on $x$ and $y$.

Let $A$ be an alternating circuit in $G$. Suppose first that $A$ meets both $H-\{x\}$ and $K-\{y\}$. Let $A_{H}=A \cap E(H)$ and $A_{K}=A \cap E(K)$. Note that $A_{H} \cup\{x\}$ and $A_{K} \cup\{y\}$ are alternating in $H$ and $K$ respectively, and hence clockwise odd. Therefore $A$ is clockwise odd.

Now suppose that $A \subseteq E(K)$. We show that $A$ is alternating in $K$. Since $A$ is alternating in $G$, we can write $A=f+g$ for 1-factors $f$ and $g$ in $G$. If $(f-A) \cap E(K)$ is a 1 -factor of $K-V(A)$, then we are done. 
Otherwise $[(f-A) \cap E(K)] \cup\{y\}$ is a 1-factor of $K-V(A)$. In both cases $A$ is alternating in $K$ and hence clockwise odd. Thus $G$ is Pfaffian.

If $G$ is obtained by splicing 1-factor covered Pfaffian graphs $H$ and $K$, then $G$ is not necessarily Pfaffian. However we proceed to describe a condition on the splice of $G$ that guarantees the desired conclusion. A cut $X$ in a graph $G$ is defined to be a minimal set of edges such that $G-X$ has more components than has $G$. An example is a splice of $G$. A cut $X$ is called tight if $|f \cap X|=1$ for every 1 -factor $f$. The required condition is tightness of the splice.

First we require the following lemma.

Lemma. Let $T$ be a spanning forest of a Pfaffian graph $G$. Then an arbitrary orientation of $T$ may be extended to a Pfaffian orientation of $G$.

Proof. We may assume without loss of generality that $G$ is connected and that $T$ is a spanning tree with a root vertex $r$, chosen arbitrarily. Let $G^{*}$ be a Pfaffian orientation of $G$, and let $X$ be the set of edges of $T$ whose orientations in $G^{*}$ and the given orientation of $T$ are distinct. For each vertex $v$, let $s(v)$ be the number of edges of $X$ in the path in $T$ joining $r$ and $v$. Thus if an edge $e$ in $T$ joins vertices $y$ and $z$, then $s(y)$ and $s(z)$ have opposite parity if and only if $e \in X$. Let $S$ be the set of all vertices $v$ with $s(v)$ even. Change the orientation of each edge of $G$ joining a vertex of $S$ to a vertex of $V(G)-S$. The set of edges of $T$ whose orientations are changed is $X$, and so the restriction to $T$ of the new orientation of $G$ is the given orientation of $T$. Moreover the new orientation of $G$ is Pfaffian, because in any alternating circuit the number of edges whose orientations are changed is even.

THEOREM 4. Let $H, K$ be 1-factor covered graphs spliced at vertices $v$, $w$ respectively to form $G$. Suppose that in $G$ the splice is tight. If $H, K$ are Pfaffian, then so is $G$.

Proof. The previous lemma shows that $H$ has a Pfaffian orientation in which each edge incident on $v$ is directed to $v$. Similarly $K$ has a Pfaffian orientation in which each edge incident on $w$ is directed from $w$. We take as our orientation of $G$ that induced by the orientations of $H$ and $K$, and show that it is Pfaffian.

Let $A$ be an alternating circuit of $G$.

Suppose $A$ does not meet the splice $C$. Without loss of generality, we may then assume that $A$ is a circuit in $K$. Note that $G-V(A)$ has a 1factor $f$, and $f$ contains exactly one edge $e$ of $C$. Let $e$ join vertices 
$v_{H} \in V(H)$ and $w_{K} \in V(K)$, and let $e_{K}$ be the edge of $E(K)$ joining $w_{K}$ and $w$. Then $(f \cap E(K)) \cup\left\{e_{K}\right\}$ is a 1-factor of $K-V(A)$. Hence $A$ is an alternating circuit in $K$, and therefore clockwise odd.

Now suppose that $A$ meets $C$. Since $C$ is tight, we have $|A \cap C|=2$. Let $A \cap C=\left\{e_{1}, e_{2}\right\}$. For each $i \in\{1,2\}$, let $e_{i}$ join vertices $v_{i} \in V(H)$ and $w_{i} \in V(K)$, let $e_{i}^{H}$ join $v_{i}$ and $v$ in $H$, and let $e_{i}^{K}$ join $w_{i}$ and $w$ in $K$. Therefore $(A \cap E(H)) \cup\left\{e_{1}^{H}, e_{2}^{H}\right\}$ and $(A \cap E(K)) \cup\left\{e_{1}^{K}, e_{2}^{K}\right\}$ are circuits. We denote them by $A_{H}$ and $A_{K}$ respectively. Again $G-V(A)$ has a 1-factor $f$, and $f \cap E(H)$ and $f \cap E(K)$ are 1-factors of $H-V\left(A_{H}\right)$ and $K-V\left(A_{K}\right)$ respectively. Hence $A_{H}$ and $A_{K}$ are alternating circuits in $H$ and $K$ respectively. Therefore both are clockwise odd. We infer that $A \cap E(H)$ has an even number of edges oriented in each sense of $A$, as does $A \cap E(K)$. Therefore $A$ is again clockwise odd.

Counterexamples exist if the assumption of the tightness of the splice is dropped. (For instance, the Petersen graph can be constructed by an appropriate splice of two wheels, each with six vertices, at their hubs. The wheels are 1-factor covered and Pfaffian, but the Petersen graph is non-Pfaffian.) However it is easy to check that, if the brick decomposition procedure is reversed, then all splices are tight.

\section{Acknowledgements}

This work was undertaken while the second author was a visitor at Massey University. He thanks its Department of Mathematics and Statistics for their kind hospitality. He also thanks the Massey University Council, the New Zealand Mathematical Society and the Austrian Science Foundation (Project S32/01) for financial assistance.

Both authors thank the referee for helpful comments, and especially for simplifying the proof of the lemma.

\section{References}

[1] P. W. Kasteleyn, 'Graph theory and crystal physics', in Graph theory and theoretical physics, pp. 43-110, (Academic Press, New York, 1967).

[2] C. H. C. Little, 'Kasteleyn's theorem and arbitrary graphs', Canad. J. Math. 25 (1973), 758-764.

[3] L. Lovász, 'On the structure of factorizable graphs,' Acta Math. Acad. Sci. Hungar. 23 (1972), 179-195. 
[4] L. Lovász, 'Matching structure and the matching lattice', J. Combin. Theory Ser. B 43 (1987), 187-222.

[5] L. Lovász and M. D. Plummer, 'On bicritical graphs,' in Infinite and finite sets, pp. 1051-1079, (Colloq. Math. Soc. J. Bolyai, No. 10, North-Holland, Amsterdam, 1975).

[6] L. Lovász and M. D. Plummer, Matching theory, (Akadémiai Kiadó, Budapest, 1986).

Massey University

Palmerston North

New Zealand
Technische Universität Graz

Kopernikusgasse 24

A-8010 Graz

Austria 